\title{
Correspondence
}

Editor: Greg Wilkinson

Contents: Supportive psychotherapy: a contradiction in terms?/Screening for HIV/Therapeutic factors in in-patient psychotherapy groups/Assaults on staff by psychiatric in-patients/Psychotherapy and dysmorphophobia/ECT for depression in dementia/ Dangerous delusions: violence and the misidentification syndromes/The need to compare the effectiveness of antidepressant drugs: in which patient populations?/Barking mad/The dopamine hypothesis/ Imipramine versus phenelzine in melancholias and dysthymic disorders/The motor disorders of severe psychiatric illness: a conflict of paradigms/Quinine psychosis/Bulimia nervosa in an atypical setting: case report from Nigeria/Catatonia in a 90 -year-old patient after depot pipothiazine injection/Mania following bereavement in a mentally handicapped man/ Psychiatric Munchausen's syndrome: a College register?/Migraine, insomnia and reactive depression due to brain serotonin deficiency?

\section{Supportive Psychotherapy: A Contradiction in Terms?}

SIR: I am writing in response to Dr Crown (Journal, February 1988, 152, 266-269). I would like to suggest that no therapy of any kind can start unless some sort of 'working alliance' or 'secure base' is first established. If one can be it is, ipso facto, supportive and an essential ingredient of dynamic psychotherapy. There is no paradox, only a puzzle that one could be perceived.

\section{Stockwell Centre Practice \\ $10 B$ Northgate Street \\ Colchester, Essex CO1 1HA}

\section{J. LuCAS}

SIR: Sidney Crown's interesting article reached a surprising conclusion. Surely all psychotherapies are, or should be, supportive? Psychotherapy is a broad term which encompasses treatments ranging from psychoanalysis to cognitive and behavioural therapies and action techniques such as Gestalt and psychodrama. The difference between the so-called 'supportive' psychotherapy and 'dynamic' psychotherapy is not in the supportive aspect, but in the aim of therapy. In the former, this is to strengthen and supplement existing defence mechanisms, leaving the unconscious alone and concentrating, as he says, on symptoms and on the present time. In dynamic psychotherapy, the aim is to make fundamental changes in these mechanisms, which involves understanding how they have arisen, on the part of the patient, through his unconscious being made conscious. The mobilising of negative emotions mentioned by Dr Crown can only be done effectively in the context of a supportive relationship. What patient would risk acknowledging his more negative side in a relationship felt to be unsupportive?

Perhaps we could replace the word 'supportive' by another such as 'maintaining' or 'non-challenging' or 'non-interpretative'?

As to the specific technical interventions as opposed to non-specific factors, Buckley et al found that interpretation and insight were key factors in symptom alleviation and positive character change, and that technical procedures related to the management of transference phenomena were critical to a positive outcome. They concluded that specific technical procedures are "central to a positive therapeutic change resulting from psychoanalysis or intensive psychotherapy, provided that they are implemented within the context of what the patient experiences as a positive therapeutic relationship".

SOPHIA HARTLAND

St Ann's Hospital

Nottingham

\section{Reference}

Buckley, P., Karasu, T. B. \& Charles, E. (1981) Psychotherapists view their personal therapy. Psychotherapy Theory Research and Practice, 18, 299-305.

SIR: I would not argue with the bulk of what Dr Crown has to say in describing dynamic and supportive psychotherapies, but I would see these as a continuum rather than separate entities. Clinical practice does not generate many patients whose treatment fits fully into one of these categories. In an individual patient the bias may be towards dynamic work where interpretations are frequent, defences challenged, 